University of Massachusetts Amherst

From the SelectedWorks of Dimitrios Maroudas

2009

\title{
Comparative Study of the Mechanical Behavior Under Biaxial Strain of Prestrained Face-centered Cubic Metallic Ultrathin Films
}

Dimitrios Maroudas, University of Massachusetts - Amherst

M. R Gungor

K. Kolluri 


\section{AIP Appled Phisics Letters \\ Comparative study of the mechanical behavior under biaxial strain of prestrained face-centered cubic metallic ultrathin films}

Kedarnath Kolluri, M. Rauf Gungor, and Dimitrios Maroudas

Citation: Appl. Phys. Lett. 94, 101911 (2009); doi: 10.1063/1.3093676

View online: http://dx.doi.org/10.1063/1.3093676

View Table of Contents: http://apl.aip.org/resource/1/APPLAB/v94/i10

Published by the American Institute of Physics.

\section{Related Articles}

Mechanism for atmosphere dependence of laser damage morphology in $\mathrm{HfO} 2 / \mathrm{SiO} 2$ high reflective films J. Appl. Phys. 112, 023111 (2012)

Superhard behaviour, low residual stress, and unique structure in diamond-like carbon films by simple bilayer approach

J. Appl. Phys. 112, 023518 (2012)

Ultrafast photoinduced mechanical strain in epitaxial BiFeO3 thin films

Appl. Phys. Lett. 101, 041902 (2012)

Mechanical and piezoresistive properties of thin silicon films deposited by plasma-enhanced chemical vapor deposition and hot-wire chemical vapor deposition at low substrate temperatures

J. Appl. Phys. 112, 024906 (2012)

Formation of stress-controlled, highly textured, $\alpha-\mathrm{SiC}$ thin films at $950^{\circ} \mathrm{C}$

J. Appl. Phys. 112, 013535 (2012)

\section{Additional information on Appl. Phys. Lett.}

Journal Homepage: http://apl.aip.org/

Journal Information: http://apl.aip.org/about/about_the_journal

Top downloads: http://apl.aip.org/features/most_downloaded

Information for Authors: http://apl.aip.org/authors

\section{ADVERTISEMENT}

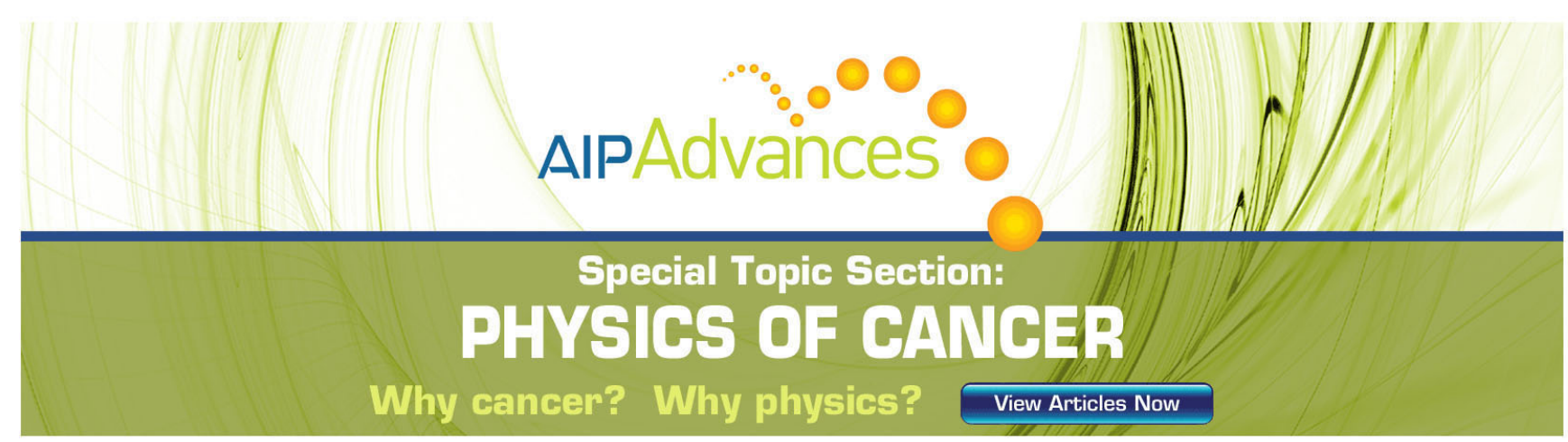




\title{
Comparative study of the mechanical behavior under biaxial strain of prestrained face-centered cubic metallic ultrathin films
}

\author{
Kedarnath Kolluri, M. Rauf Gungor, and Dimitrios Maroudas ${ }^{a}$ \\ Department of Chemical Engineering, University of Massachusetts Amherst, Amherst, \\ Massachusetts 01003-3110, USA
}

(Received 12 January 2009; accepted 10 February 2009; published online 11 March 2009)

\begin{abstract}
We report a molecular-dynamics study of the mechanical response to dynamic biaxial tensile straining of nanometer-scale-thick $\mathrm{Al}, \mathrm{Cu}$, and $\mathrm{Ni}$ films. We find that the mechanical behavior of such films of face-centered cubic metals with moderate-to-high propensity for stacking-fault formation $(\mathrm{Cu}$ and $\mathrm{Ni})$ is significantly different from those where such propensity is low $(\mathrm{Al})$. The plastic strain rate in $\mathrm{Cu}$ and $\mathrm{Ni}$ films is greater than that in $\mathrm{Al}$ ones, leading to an extended easy-glide stage in $\mathrm{Cu}$ and $\mathrm{Ni}$ but not in $\mathrm{Al}$ films. These differences arise due to the different dislocation annihilation mechanisms in the two film categories. (c) 2009 American Institute of Physics.

[DOI: $10.1063 / 1.3093676$ ]
\end{abstract}

A broad range of modern technologies relies increasingly on the use of nanometer-scale-thick metallic films. During the numerous processes involved in the development of micro- and nanoscale devices, the constituent metallic materials are subjected to various thermomechanical environments, which cause their straining and plastic deformation. The plastic deformation of single-crystalline metallic materials is facilitated by nucleation and glide of dislocations, as well as interactions of dislocations with various other defects. In nanostructured forms of metals, however, such plastic deformation phenomena are not well understood. Metallic nanostructures and other small-volume structures exhibit, for example, ultrahigh strength unlike their bulk counterparts. ${ }^{1,2}$ In these structures, dislocation depletion is responsible for the observed ultrahigh strength. ${ }^{2}$ For example, in compression experiments of Ni single-crystalline nanopillars with a high initial dislocation density, the dislocation density always decreased during the application of strain; ${ }^{3}$ subsequently, the nearly defect-free crystal deformed elastically until additional dislocations nucleated.

In metallic ultrathin films and other nanostructures, the underlying atomic-scale deformation mechanisms are particularly difficult to study experimentally. The purpose of this article is a comparative atomic-scale analysis of the dynamic deformation of freestanding $\mathrm{Al}, \mathrm{Cu}$, and $\mathrm{Ni}$ ultrathin films based on large-scale molecular-dynamics (MD) simulations. Our analysis reveals that dislocation annihilation is more prevalent in films of metals characterized by a high propensity for the formation of stacking faults. The different mechanisms of dislocation interaction and annihilation account for the differences in the mechanical behavior between films of metals with more stacking faults (such as $\mathrm{Cu}$ and $\mathrm{Ni}$ ) and those with fewer stacking faults (such as Al).

In our MD simulations, we employed slab supercells consisting of freestanding single-crystalline films with periodic boundary conditions applied in the $x$ - and $y$-directions in a Cartesian representation; the film planes were oriented normal to the $z$ axis and the films had thicknesses of a few nanometers. The Cartesian $x, y$, and $z$ axes were taken along

\footnotetext{
${ }^{\text {a) }}$ Author to whom correspondence should be addressed. Electronic mail: maroudas@ecs.umass.edu.
}

the $[\overline{1} 10],[\overline{1} \overline{1} 2]$, and $[111]$ crystallographic directions, respectively, with the film plane corresponding to the (111) crystallographic plane. For the results reported here, we employed simulation cells that contained 1520640 atoms with edge sizes of $240 d_{x}, 432 d_{y}$, and $21 d_{z}$ in the $x$ -,$y$-, and $z$-directions, respectively, in the films' unstrained state; $d_{x}$, $d_{y}$, and $d_{z}$ are the equilibrium interplanar spacings of the crystals in the [110], [1̄12], and [111] crystallographic directions, respectively. The films were subjected to dynamic biaxial tensile straining at a constant high strain rate of $7 \times 10^{8} \mathrm{~s}^{-1}$ and constant temperature, $T=100 \mathrm{~K}$. We used the public-domain computer software LAMMPS (Ref. 4) and ATOMEYE (Ref. 5) for the MD simulations and the visualization of the MD-generated atomic configurations, respectively, and common neighbor analysis ${ }^{6}$ for detailed structural characterization. Our samples were prepared (prestrained) according to the techniques of Ref. 7; the initial dislocation densities in our samples (after pretreatment) ranged from 1.2 to $1.8 \times 10^{17} \mathrm{~m}^{-2}$. A detailed description of our MD simulation methods and the methods of analysis of the simulation results can be found in the supplementary document. ${ }^{8}$

The three different fcc metals examined in our study are characterized by different propensities for formation of stacking faults; this propensity is expressed by the ratio $\gamma_{s} / \gamma_{u}$, where $\gamma_{s}$ and $\gamma_{u}$ are the stable and unstable stackingfault energies, respectively; high (low) values of $\gamma_{s} / \gamma_{u}$ imply small (large) total stacking-fault area. In our study, the interatomic interactions were described using embedded-atommethod (EAM) potentials. The parametrization developed by Mishin et al. was used for $\mathrm{Al}$ (Ref. 9) and $\mathrm{Cu},{ }^{10}$ whereas that developed by Angelo et al. was used for Ni. ${ }^{11}$ The choice of the EAM potential for each material was based on its accuracy to predict $\gamma_{s}, \gamma_{u}$, and the ideal critical shear stress in comparison with the predictions of ab initio calculations, ${ }^{12,13}$ as shown in Table I.

The mechanical responses of the three different metallic films examined in this study are shown in Fig. 1. Figures 1(a) and 1(b) show the evolution of the von Mises shear stress (calculated using the Virial formula ${ }^{14}$ ) as a fraction of the material's ideal shear strength, $\sigma_{\mathrm{vM}} / \sigma_{u}$, and of the dislocation density as a fraction of the initial dislocation density, 
TABLE I. Stable and unstable stacking-fault energies, $\gamma_{s}$ and $\gamma_{u}$, respectively, and ideal simple shear strength for $\mathrm{Al}, \mathrm{Ni}$, and $\mathrm{Cu}$ according to different EAM potentials; the EAM values (in boldface) are compared with previously published values from first-principles calculations (in italics).

\begin{tabular}{|c|c|c|c|c|}
\hline Metal & $\begin{array}{c}\gamma_{u} \\
\left(\mathrm{~mJ} / \mathrm{m}^{2}\right)\end{array}$ & $\begin{array}{c}\gamma_{s} \\
\left(\mathrm{~mJ} / \mathrm{m}^{2}\right)\end{array}$ & $\gamma_{s} / \gamma_{u}$ & $\begin{array}{l}\text { Ideal shear strength } \\
\qquad(\mathrm{GPa})\end{array}$ \\
\hline $\mathrm{Al}$ & $\begin{array}{l}195^{\mathrm{a}} \\
178^{\mathrm{b}}\end{array}$ & $\begin{array}{c}158.9^{\mathrm{a}} \\
146^{\mathrm{b}}\end{array}$ & $\begin{array}{l}\mathbf{0 . 8 2} \\
0.83^{\mathrm{a}}\end{array}$ & $\begin{array}{l}\mathbf{3 . 8 1 ^ { \mathrm { a } }} \\
3.73^{\mathrm{c}}\end{array}$ \\
\hline $\mathrm{Ni}$ & $\begin{array}{c}\mathbf{2 6 3 . 8}^{\mathrm{d}} \\
278^{\mathrm{b}}\end{array}$ & $\begin{array}{c}\mathbf{1 2 4 . 7 ^ { \mathrm { d } }} \\
137^{\mathrm{b}}\end{array}$ & $\begin{array}{l}\mathbf{0 . 4 7 ^ { \mathrm { d } }} \\
0.49^{\mathrm{b}}\end{array}$ & $\begin{array}{l}\mathbf{5 . 8 8 ^ { \mathrm { d } }} \\
6.29^{\mathrm{c}}\end{array}$ \\
\hline $\mathrm{Cu}$ & $\mathbf{1 8 1 . 1}^{\mathrm{e}}$ & $\begin{array}{l}44.4^{\mathrm{e}} \\
38^{\mathrm{b}}\end{array}$ & $\begin{array}{l}\mathbf{0 . 2 5} \\
0.23^{\mathrm{b}}\end{array}$ & $\begin{array}{l}3.97^{\mathrm{e}} \\
3.45^{\mathrm{c}}\end{array}$ \\
\hline
\end{tabular}

${ }^{\mathrm{a}}$ Reference 9.

${ }^{\mathrm{b}}$ Reference 13.

${ }^{\mathrm{c}}$ Reference 12 .

$\rho / \rho_{0}$, respectively, during the dynamic biaxial straining of the three $(\mathrm{Al}, \mathrm{Cu}$, and $\mathrm{Ni}$ ) thin films; the applied strain rate is constant and the applied strain level, $\varepsilon$, increases linearly with time until failure. In our simulations, the maximum $\sigma_{\mathrm{vM}}$ for the different metallic films varied between $35.2 \%$ and $44.5 \%$ of their ideal simple shear strength according to the EAM models employed. These maximum $\sigma_{\mathrm{vM}}$ values are consistent with experimental measurements on Au nanopillars that reached $44 \%$ of their theoretically predicted ideal pure shear strength. ${ }^{15}$ Figure 1(c) shows the evolution of the plastic strain rate, $\dot{\gamma}=\sum_{i}\left(b_{i} l_{i} \dot{x}_{i} / V\right)=\sum_{i}\left(b_{i} \dot{S}_{i} / V\right)$, where $V$ is the volume of the thin film and $b_{i}, l_{i}$, and $\dot{x}_{i}$ are the Burgers vector of, length of, and distance swept by the $i$ th gliding dislocation over a specified time interval, respectively; $\dot{S}_{i}$ $=l_{i} \dot{x}_{i}$ is the area swept by a dislocation of length $l_{i}$ over the same time interval.

From the stress-strain curves of Fig. 1(a), it is evident that the mechanical response of the $\mathrm{Al}$ film is significantly different from that of the $\mathrm{Ni}$ and $\mathrm{Cu}$ films. In the initial deformation stage (stage I), $\sigma_{\mathrm{vM}}$ increases in all three metallic films. Beyond this stage, in the $\mathrm{Ni}$ and $\mathrm{Cu}$ films, we observe an extended "easy-glide" stage (stage II) where the stress remains approximately constant; this easy-glide stage is then followed by a stage of stress buildup (stage III) in the films. In $\mathrm{Al}$, however, $\sigma_{\mathrm{vM}}$ increases monotonically during stage II. As shown in Fig. 1(b), the dislocation density decreases for all three films until the stress buildup is high enough to cause nucleation of additional dislocations; nevertheless, in the $\mathrm{Al} \mathrm{film,} \mathrm{during} \mathrm{stage} \mathrm{II,} \mathrm{the} \mathrm{dislocations} \mathrm{are}$ annihilated at a slower rate compared to that in the $\mathrm{Ni}$ and $\mathrm{Cu}$ films, and at the end of stage II, the dislocation density, $\rho / \rho_{0}$, is much higher than those in the $\mathrm{Ni}$ and $\mathrm{Cu}$ films. As shown in Fig. 1(c), for all three films, the plastic strain rate increases during stage I; however, the increase is $\sim 60 \%$ greater for the $\mathrm{Cu}$ and $\mathrm{Ni}$ films compared to that in the $\mathrm{Al}$ film. Then, $\dot{\gamma}$ decreases in the $\mathrm{Cu}$ and $\mathrm{Ni}$ films until additional dislocations are nucleated from the surface, whereas it remains nearly constant in the Al film. With nucleation of additional dislocations in stage III, $\dot{\gamma}$ increases again for all three films. Interestingly, the decrease in $\dot{\gamma}$ in the $\mathrm{Cu}$ and $\mathrm{Ni}$ films to levels comparable to those in the Al film marks the end of the easy-glide stage in the $\mathrm{Cu}$ and $\mathrm{Ni}$ films.

In Figs. 1(a)-1(c), the films' mechanical response during stage I demonstrates that the increase in the von Mises stress, $\sigma_{\mathrm{vM}}$, corresponds to the mobilization of the initial dislocation population. In stage I, the plastic strain rate increases more in the $\mathrm{Ni}$ and $\mathrm{Cu}$ films than in the $\mathrm{Al}$ film, indicating higher dislocation mobility in films of metals with low-to-moderate values of $\gamma_{s} / \gamma_{u}$ than in those with high values of $\gamma_{s} / \gamma_{u}$. In the $\mathrm{Cu}$ and $\mathrm{Ni}$ films, the high plastic flow rate leads to the dissipation of the stress imparted in the films due to the application of biaxial strain, causing $\sigma_{\mathrm{vM}}$ in these films to remain nearly constant during stage II. On the other hand, the higher dislocation mobility in the $\mathrm{Ni}$ and $\mathrm{Cu}$ films promotes interactions between dislocations resulting in greater dislocation annihilation rates in these films than that observed in the $\mathrm{Al}$ film. In the $\mathrm{Cu}$ and $\mathrm{Ni}$ films, this dislocation annihilation process causes the plastic flow rate to decrease with increasing applied biaxial strain during stage II [Fig. $1(\mathrm{c})]$. In contrast, during stage II, the plastic flow rate in the Al thin films remains essentially constant and lower than those in the $\mathrm{Ni}$ and $\mathrm{Cu}$ films. Due to the fewer interactions between dislocations in the $\mathrm{Al}$ film, the dislocation annihilation rate in this film is nearly half as those in the $\mathrm{Ni}$ and $\mathrm{Cu}$ films. Additionally, the stress dissipation in the $\mathrm{Al}$ film is lower due to the lower plastic flow rate, and $\sigma_{\mathrm{vM}}$ in the $\mathrm{Al}$ film increases monotonically in stage II [Fig. 1(a)].

At the onset of stage III, the plastic flow rates in the $\mathrm{Ni}$ and $\mathrm{Cu}$ films are reduced below that of the $\mathrm{Al}$ thin film and the stress in the $\mathrm{Ni}$ and $\mathrm{Cu}$ films starts to increase. During stage III [Fig. 1(a)], for the $\mathrm{Ni}$ and $\mathrm{Cu}$ films, the increasing stress is accompanied by a very low dislocation density (up to about $20 \%$ of $\rho_{0}$ ) in these films; the occurrence of such low dislocation densities can be characterized as "dislocation starvation." In the Al film, however, the limited plastic flow does not promote interactions between dislocations and, con-
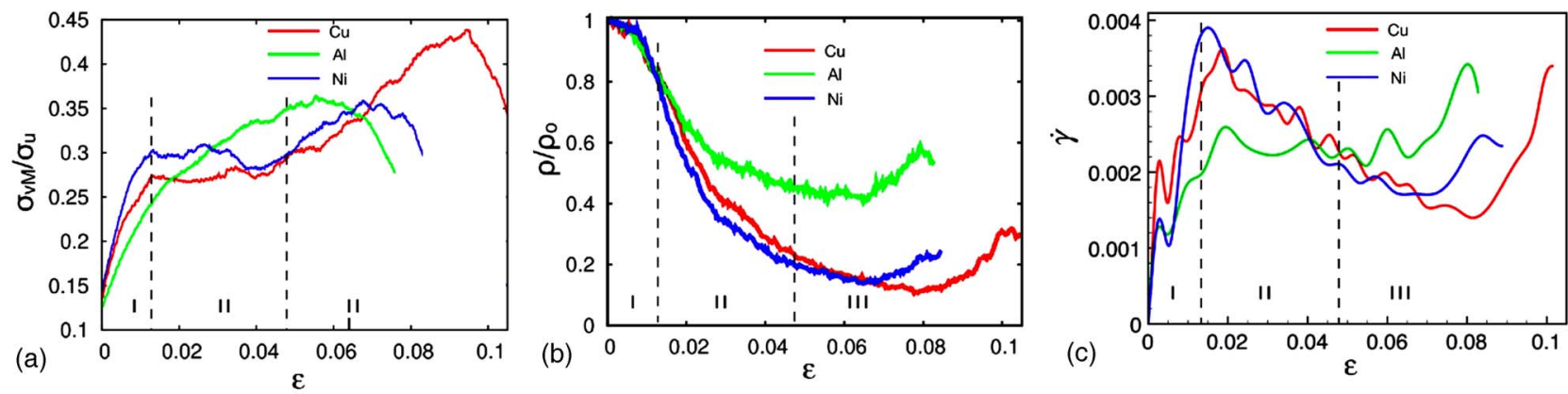

FIG. 1. (Color online) Evolution (i.e., dependence on strain, $\varepsilon$, during dynamic deformation) of the (a) von Mises stress, $\sigma_{\mathrm{vM}}$, as a fraction of the ideal shear strength, $\sigma_{u}$, (b) dislocation density, $\rho$, as a fraction of the initial dislocation density, $\rho_{0}$, and (c) plastic strain rate, $\dot{\gamma}$, from MD simulation of dynamic biaxial tensile straining of nanometer-scale-thick films of $\mathrm{Al}, \mathrm{Cu}$, and $\mathrm{Ni}$ [light gray (green online), gray (red online), and dark gray (blue online) lines, respectively]. 


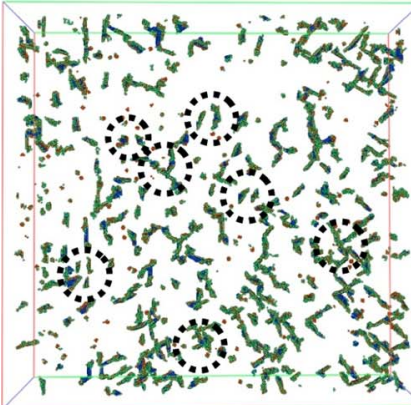

(a) $\varepsilon=0 \%$

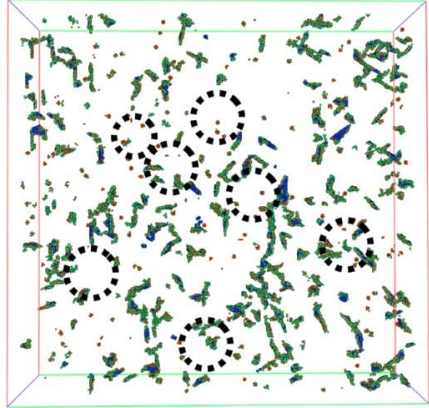

(b) $\varepsilon=3.0 \%$

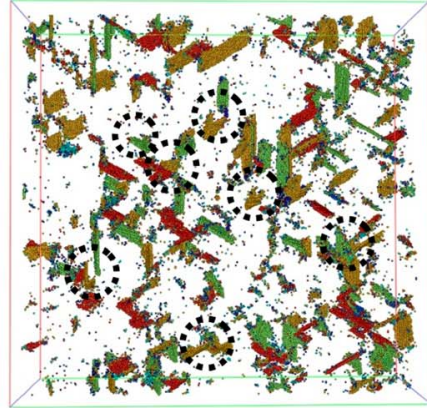

(c) $\varepsilon=3.0 \%$

FIG. 2. (Color online) Top view of the Al film under applied biaxial strains of (a) $\varepsilon=0$ and (b) $\varepsilon=3 \%$. Light green and orange colored atoms form the dislocation cores, while atoms in stacking faults are colored blue; atoms in a locally perfect fcc environment and surface atoms are not shown for clarity. Collinear dislocation pairs that are marked (in circles) in (a) have been annihilated in (b). (c) Top view of the Al film in (b) with atoms colored according to their slip vector (analogous to the Burgers vector). For clarity, only atoms that have slipped with Burgers vectors $\mathbf{b}=(a / 2)[101],(a / 2)[\overline{1} 10]$, and $(a / 2)[011]$ are shown and colored green, gold, and red, respectively. For interpretation of the references to color in this figure legend, the reader is referred to the online version of this article.

sequently, the minimum dislocation density (as a fraction of $\left.\rho_{0}\right)$ in this film is more than twice as high as that in the $\mathrm{Ni}$ and $\mathrm{Cu}$ films [Fig. 1(b)].

In $\mathrm{Cu}$ and $\mathrm{Ni}$ thin films, Shockley partial dislocations and stacking faults are more prevalent than perfect dislocations, and stacking faults act as sources for dislocation cross slip and eventual dislocation annihilation. These stackingfault-mediated mechanisms of dislocation dissociation and annihilation have been analyzed extensively elsewhere; ${ }^{16}$ they are similar to the Friedel-Escaig ${ }^{17}$ and Fleischer-type ${ }^{18}$ cross-slip mechanisms observed in bulk and small-volume structures of fcc metals. The mechanisms of dislocation annihilation in $\mathrm{Al}$ thin films, however, are primarily due to interactions between perfect dislocations with opposite Burgers vectors in different glide planes. Figure 2 shows top views at different time instants of the Al film that exhibited the mechanical response shown in Fig. 1. Figure 2(a) shows the initial microstructure of the film. In Figs. 2(a) and 2(b), blue and other colored atoms denote atoms in a locally perfect hexagonal close-packed environment and atoms in dislocation cores, respectively. In Fig. 2(c), atoms that have slipped due to the glide motion of perfect dislocations are shown; each color corresponds to a specific Burgers vector. For clarity, only three Burgers vectors, namely, $\mathbf{b}=(a / 2)$ $\times[101],(a / 2)[\overline{1} 10]$, and $(a / 2)[011]$ are included and other atoms are not shown; for example, the golden colored atoms correspond to those that slipped due to the glide of dislocations with $\mathbf{b}=(a / 2)[\overline{1} 10]$. In Figs. 2(a)-2(c), multiple locations are marked to show clearly dislocations with opposite Burgers vectors in different glide planes that interact and annihilate one another. The underlying dislocation annihilation mechanisms ${ }^{8}$ are completely analogous to collinear interactions that have been demonstrated in bulk fcc metals. ${ }^{19}$ These differences in the films' dislocation population evolution and the mechanisms of dislocation depletion suggest that stacking faults play a significant role in the annihilation of dislocations in ultrathin films of fcc metals with moderateto-high propensity for stacking-fault formation.

In summary, our MD simulations of various ultrathin fec metallic films under dynamic biaxial tensile straining indicate that there are significant differences in the evolution of the flow stress between films of metals with high propensity for formation of stacking faults and those with low such propensity. We find that the plastic flow in $\mathrm{Ni}$ and $\mathrm{Cu}$ films is far more pronounced than in $\mathrm{Al}$ ones during the initial stages of dynamic deformation, causing significant stress dissipation and an extended easy-glide stage in $\mathrm{Cu}$ and $\mathrm{Ni}$ thin films; we attribute these differences to the different mechanisms of dislocation interaction and annihilation in the $\mathrm{Cu}$ and $\mathrm{Ni}$ films from those in the $\mathrm{Al}$ films. Our analysis provides further insights into the subtle differences in the mechanical behavior of thin films of fcc metals and the underlying mechanisms of dislocation depletion.

This work was supported by the Office of Basic Energy Sciences, U.S. Department of Energy through Grant No. DEFG02-07ER46407. Supercomputing facilities were made available by the National Science Foundation through TeraGrid resources provided by NCSA.

${ }^{1}$ S. S. Brenner, Science 128, 569 (1958).

${ }^{2}$ J. R. Greer and W. D. Nix, Phys. Rev. B 73, 245410 (2006).

${ }^{3}$ Z. W. Shan, R. K. Mishra, S. A. S. Asif, O. L. Warren, and A. M. Minor, Nature Mater. 7, 115 (2008).

${ }^{4}$ S. Plimpton, J. Comput. Phys. 1, 117 (1995).

${ }^{5}$ J. Li, Modell. Simul. Mater. Sci. Eng. 11, 173 (2003).

${ }^{6}$ J. D. Honeycutt and H. C. Andersen, J. Phys. Chem. 91, 4950 (1987).

${ }^{7}$ K. Kolluri, M. R. Gungor, and D. Maroudas, J. Appl. Phys. 103, 123517 (2008).

${ }^{8}$ See EPAPS Document No. E-APPLAB-94-105909 for a detailed description of our MD simulation methods and the methods of analysis of the simulation results. For more information on EPAPS, see http:// www.aip.org./pubservs/epaps.html.

${ }^{9}$ Y. Mishin, D. Farkas, M. J. Mehl, and D. A. Papaconstantopoulos, Phys. Rev. B 59, 3393 (1999).

${ }^{10}$ Y. Mishin, M. J. Mehl, D. A. Papaconstantopoulos, A. F. Voter, and J. D. Kress, Phys. Rev. B 63, 224106 (2001).

${ }^{11}$ J. E. Angelo, N. R. Moody, and M. I. Baskes, Modell. Simul. Mater. Sci. Eng. 3, 289 (1995).

${ }^{12}$ S. Ogata, J. Li, N. Hirosaki, Y. Shibutani, and S. Yip, Phys. Rev. B 70, 104104 (2004).

${ }^{13}$ C. Brandl, P. M. Derlet, and H. Van Swygenhoven, Phys. Rev. B 76, 054124 (2007).

${ }^{14}$ A. M. Allen and D. J. Tildesley, Computer Simulation of Liquids (Oxford University Press, Oxford, 1990).

${ }^{15}$ S. Brinckmann, J. Y. Kim, and J. R. Greer, Phys. Rev. Lett. 100, 155502 (2008).

${ }^{16}$ K. Kolluri, M. R. Gungor, and D. Maroudas, arXiv:0901.1092v1.

${ }^{17}$ B. Escaig, J. Phys. (Paris) 29, 225 (1968).

${ }^{18}$ R. L. Fleischer, Acta Metall. 7, 134 (1959).

${ }^{19}$ R. Madec, B. Devincre, L. Kubin, T. Hoc, and D. Rodney, Science 301, 1879 (2003) 